\title{
Effects of irregular two-dimensional and three-dimensional surface roughness in turbulent channel flows
}

\author{
M. De Marchis ${ }^{a, *}$, E. Napoli ${ }^{b}$ \\ ${ }^{a}$ Facoltà di Ingegneria e Architettura, Università degli Studi di Enna "Kore", Cittadella Universitaria, Enna, Italy \\ ${ }^{\mathrm{b}}$ Dipartimento di Ingegneria Civile, Ambientale e Aerospaziale, Università degli Studi di Palermo, Viale delle Scienze, Palermo, Italy
}

\section{A R T I C L E I N F O}

\section{Article history:}

Received 29 December 2011

Received in revised form 27 March 2012

Accepted 7 April 2012

Available online 8 May 2012

\section{Keywords:}

Turbulence simulation

Channel flow

LES

Three-dimensional irregular roughness

\begin{abstract}
A B S T R A C T
Wall-resolved Large Eddy Simulation of fully developed turbulent channel flows over two different rough surfaces is performed to investigate on the effects of irregular 2D and 3D roughness on the turbulence. The two geometries are obtained through the superimposition of sinusoidal functions having random amplitudes and different wave lengths. In the 2D configuration the irregular shape in the longitudinal direction is replicated in the transverse one, while in the 3D case the sinusoidal functions are generated both in streamwise and spanwise directions. Both channel walls are roughened in such a way as to obtain surfaces with statistically equivalent roughness height, but different shapes. In order to compare the turbulence properties over the two rough walls and to analyse the differences with a smooth wall, the simulations are performed at the same Reynolds number $R e_{\tau}=395$. The same mean roughness height $h=0.05 \delta$ ( $\delta$ the half channel height) is used for the rough walls.

The roughness function obtained with the 3D roughness is larger than in the 2D case, although the two walls share the same mean height. Thus, the considered irregular 3D roughness is more effective in reducing the flow velocity with respect to the $2 \mathrm{D}$ roughness, coherently with the literature results that identified a clear dependence of the roughness function on the effective slope (see Napoli et al. (2008)), higher in the generated 3D rough wall. The analysis of higher-order statistics shows that the effects of the roughness, independently on its two- or three-dimensional shape, are mainly confined in the inner region, supporting the Townsend's wall similarity hypothesis. The tendency towards the isotropization is investigated through the ratio between the resolved Reynolds stress components, putting in light that the 3D irregular rough wall induces an higher reduction of the anisotropy, with respect to the 2D case. (c) 2012 Elsevier Inc. All rights reserved.
\end{abstract}

\section{Introduction}

Turbulent flows over rough surfaces can be encountered in a wide range of engineering applications. These include, but are not limited to, meteorological fields, hydraulic pipes, open channel flows, currents around marine vehicles or aircrafts. Both experimental and numerical researches were focused on the understanding of the effects of the roughness on turbulent flows. A recent review of these analyses can be found in Jimenez (2004).

In the studies conducted herein, the roughness has been represented in very different ways, frequently using two- or threedimensional elements having identical geometry, arranged in regular or irregular patterns. Most analyses focused the attention on the effects on the turbulent flows of two-dimensional roughness elements, regularly arranged over flat plates (see, among others, Djenidi et al. (1999), Leonardi et al. (2003), Ikeda and Durbin

\footnotetext{
* Corresponding author.

E-mail addresses: mauro.demarchis@unikore.it (M. De Marchis),enrico.napoli@ unipa.it (E. Napoli)
}

(2007) and literature therein). Nevertheless, real roughness is characterized by three-dimensional irregular geometry. Acharya et al. (1986) pointed out that the effects of two-dimensional roughness on the turbulent flows can be very different from those over real three-dimensional geometries, where the cavities between roughness elements in the streamwise and spanwise directions have strong influence. In their experiments the authors compared the effects of four rough walls: two surfaces representative of degraded turbines, a sand-cast surface and a regular mesh screen rough surface. The authors aimed at obtaining a statistical descriptor of the rough surface, not involving the equivalent sand-grain roughness parameter. Krogstad and Antonia (1999) carried out experimental analyses comparing the effects of two distinct surface roughness on the turbulent boundary layer. They considered a typical twodimensional rod roughness and a three-dimensional rough surface made up by a woven stainless steel mesh screen. The two surfaces were designed to produce the same downward shift of the mean velocity profiles in the $\log$ region (the roughness function). The experimental results put in light that analysis of only the mean velocity profiles is inadequate to completely characterize the 
effects of the surface roughness and that higher order statistics must be considered as well. The comparison between two-dimensional and three-dimensional roughness showed very different effects on the turbulent flows both in the inner and outer layers. Later, Antonia and Krogstad (2001) confirmed that different roughness geometries, having the same roughness function, can produce very different turbulence statistics. The authors, moreover, making use of the Anisotropy Invariant Map (AIM) analysis, found that three-dimensional roughness reduces the anisotropy, compared to smooth walls, more than 2D rod bars. This conclusion was further strengthened by Smalley et al. (2002), who compared the effects of 2D (square cavities and rod bars) and 3D (wire-mesh and perforated plates) rough surfaces. Tachie et al. (2003) compared the effects of sand grain and wire mesh roughness in a turbulent open channel flow. The comparison between the two rough surfaces and the smooth reference wall showed that both rough walls enhance the turbulence intensities and the Reynolds shear stress in the roughness sub-layer and in the outer layer. The authors observed too that the regular wire mesh rough surface modifies the turbulence characteristics more than the sand grain roughness. Laboratory experiments in similar rough geometries were performed by Flack et al. (2005) and Schultz and Flack (2005), whose results provided compelling support for the Townsend's similarity hypothesis (Townsend, 1976) for both the sand grain and the wire mesh roughness. Bakken et al. (2005), comparing the effects of square bars and perforated plates with those induced by mesh geometry, found that in the outer layer the roughness effect is small, further supporting the Townsend's wall similarity hypothesis. They concluded that the hypothesis can be contradicted in channels roughened on one side only, due to the asymmetry in the boundary conditions, while it holds in symmetric channels. More recently, Shah et al. (2008) compared the effects of twodimensional (transverse square bars) and three-dimensional (wire mesh) roughness, through the particle image velocimetry (PIV) technique. The authors found that wire mesh (in the transitional regime) effects are confined to the buffer region, while the rib roughness (in fully rough regime) effects extend to the entire boundary layer. They attributed this result to the high ratio between the boundary layer thickness and the equivalent sand grain roughness $k_{s}$.

In the literature cited above, wall roughness was reproduced through 2D or 3D idealized regularly arranged elements. The natural wall corrugations of environmental and industrial flows, on the other side, are generally characterized by irregular geometries, with crests and troughs of random height. Nikuradse (1933) and Colebrook (1939), in their experiments, already observed that the effects of real roughness on the turbulence can be very different from those of regular elements. Most studies on the real roughness effects on turbulent flows were conducted through experimental analyses. Bons et al. (2001) and Bons (2002) performed experimental measurements over a number of in-service turbine blades and vanes, showing that the degradation mechanisms strongly modify the skin friction and heat transfer coefficients in a different way as compared to ordered arrays of roughness elements. Further studies on the irregular surfaces of in-service gas turbine blades performed by Subramanian et al. (2004) showed that roughness increases the turbulence intensity in the inner layer, with a corresponding tendency toward isotropization. In spite of this, no roughness effect on the outer layer structure of the boundary layer was observed in their experiments. Wu and Christensen (2006) analyzed, through experimental investigation, a rough surface replicated from a surface scan of a damaged turbine blade. In-service turbine blades, in fact, are subjected to deposition of foreign material, pitting and spallation that produce non-uniform three-dimensional irregular surface conditions. The authors observed a dramatical increase of the Reynolds stress in the inner layer, as compared to the smooth-wall case, and attributed this change to the increase of intense ejections and sweeps. The analysis of their results confirmed the wall similarity hypothesis of Townsend. Successively, Wu and Christensen (2007) analyzed through PIV technique a highly irregular rough surface, quite different from the idealized geometries normally studied in the laboratory experiments. The comparison of the first- and second-order statistics and of the two-point autocorrelation in smooth and rough surfaces showed that intense differences occur in the roughness sub-layer, while a collapse of the turbulence statistics in the outer region was verified. Similar results were obtained by Connelly et al. (2006) in their experiments of turbulent flows over several rough surfaces consisting of wetdry sandpaper and wire mesh of different heights. Recently, Birch and Morrison (2011) carried out a series of experimental investigations in fully developed turbulent channel flows, comparing the effects of a smooth surface and two irregular rough surfaces, obtained using an isotropic grit and an uniform mesh of rectangular elements arranged in a diamond pattern. The authors focused the attention on the distinction between the self- and outersimilarities, through the analysis of the streamwise velocity component.

Very few studies of three-dimensional rough surfaces have been performed through numerical simulations. This can be mainly attributed to the difficulty in obtaining a detailed representation of a complex surface with computational grids. To the best knowledge of the authors, the few numerical analyses of turbulent flows over 3D rough surfaces were conducted only over regular rough geometries. Direct Numerical Simulation (DNS) of the turbulent flow over an idealized gravel bed generated with spheres of uniform size was performed by Singh et al. (2007), where the fully rough regime was considered. The simulation confirmed the wall similarity hypothesis. A similar 3D regular roughness was analyzed by Bhaganagar et al. (2006) that, simulating the effects of three rough surfaces having the same shape and different mean heights, argued that the region where inner- and outer-layer interactions occur may be affected by the size of the roughness elements. 3D roughness was also simulated by Coceal et al. (2006), who used regular arrays of urban-like, cubical obstacles of identical height over a flat plane. The analysis, carried out with the DNS technique, confirmed the need to consider three-dimensional rough surfaces to obtain realistic behaviors. Two-dimensional representations, in fact, may hide important flow structures, which could develop around three-dimensional elements. Analogous results were obtained by Bhaganagar and Hsu (2009), that performed a DNS to compare the effects of two- and three-dimensional ripples in turbulent channel flows. They found that the turbulence characteristics depend on the two- or three-dimensionality of the ripples, confirming the importance of further investigate the effects of three-dimensional roughness. Leonardi and Castro (2010) carried out a Direct Numerical Simulation of the channel flow over rough walls comprising staggered arrays of cubes, considering various plan area densities. The authors found that increasing the density of three-dimensional roughness elements leads to the decrease of the normalized vertical stresses around the top of the elements, in contrast to the observation made on two-dimensional roughness. Furthermore, they found that, for three-dimensional roughness, turbulence stresses in the outer layer are quite similar. Volino et al. (2009) attributed the difference between the effects of regular $2 \mathrm{D}$ and $3 \mathrm{D}$ roughness on the turbulence to the fact that the largest scale motions generated by three-dimensional roughness are of the order of the roughness height, while two-dimensional roughness may generate much larger scales of motion, due to the width of the roughness elements. This result was recently confirmed by Volino et al. (2011), where a direct comparison between two-dimensional transverse bars and three-dimensional transverse rows of staggered cubes was carried out. Lee et al. 
(2011) carried out a turbulent boundary layer DNS, analyzing the effects, as compared to smooth walls, of two-dimensional rodroughness and three-dimensional regularly arrayed cubes. The authors found that 3D rough surfaces cause a weaker effect than 2D wall roughness on the streamwise velocity. Xie et al. (2008) carried out a Large Eddy Simulation (LES) of the turbulent flow over random urban-like obstacles. The comparison with uniform urban-like roughness confirmed that the flow field over random irregular elements is quite different from that over regular roughness and that the flow over isolated obstacles cannot be representative of the features of turbulent flows over real rough surfaces.

As it was pointed out, in the numerical researches above analyzed only regular roughness elements were considered. Irregular rough walls ware studied by Napoli et al. (2008) and more recently by De Marchis et al. (2010) (hereafter referred to as DNA10), using the LES technique. The authors analyzed the modification of turbulence statistics and instantaneous structures in archetypal 2D irregular rough walls generated through superimposition of sinusoidal functions with random amplitudes. All the turbulent regimes were investigated. The results confirmed that the roughness dramatically modifies the turbulence in the roughness sub-layer, while the outer layer is unaffected by the specific wall configuration, irrespective of the roughness height.

The scope of the present research is to provide further insight into the effects of three-dimensional irregular rough walls on turbulent flows. Specifically, the effects of a two-dimensional irregular rough wall analyzed by DNA10 is compared with those of an equivalent irregular three-dimensional rough surface. The interaction between the inner and outer layers is also investigated, to check the validity of the Townsend's wall similarity hypothesis in turbulent flows over irregular rough surfaces.

The paper is organized as follows. Section 2 contains a description of the physical problem under investigation, the mathematical formulation herein employed and some details on the numerical method. Results are discussed in Section 3 and conclusions are drawn in Section 4.

\section{Numerical approach}

\subsection{Mathematical formulation and numerical procedure}

The turbulent channel flow is resolved using the Large Eddy Simulation approach, based on the numerical solution of the filtered Navier-Stokes equations. In the conventional summation approach, the filtered Navier-Stokes and continuity equations for incompressible fluids, read:

$\frac{\partial \bar{u}_{i}}{\partial t}+\frac{\partial \bar{u}_{i} \bar{u}_{j}}{\partial x_{j}}-\frac{1}{R e_{\tau}} \frac{\partial^{2} \bar{u}_{i}}{\partial x_{j} \partial x_{j}}+\frac{\partial \bar{p}}{\partial x_{i}}+\frac{\partial \tau_{i j}}{\partial x_{j}}+\Pi \delta_{i 1}=0$

and

$\frac{\partial \bar{u}_{i}}{\partial x_{i}}=0$

where the variables are made non-dimensional with the friction velocity $u^{*}$ and the channel half-width $\delta, x_{i}$ is the $i$ th coordinate (with $x_{1}, x_{2}$ and $x_{3}$ the streamwise, spanwise and wall-normal directions, respectively), $t$ is the time, $\bar{u}_{i}$ is the $i$ th component of the filtered velocity field, $\bar{p}$ is the filtered pressure field in kinematic units (i.e., divided by the fluid density), $\tau_{i j}=\overline{u_{i} u_{j}}-\overline{u_{i}} \overline{u_{j}}$ is the sub-grid scale turbulent stress tensor, $\operatorname{Re}_{\tau}=u^{*} \delta / v$ is the frictional Reynolds number, $\Pi$ is the imposed non-dimensional pressure gradient to drive the flow and $\delta_{i j}$ is the Kronecker function $\left(\delta_{i j}=1\right.$ for $i=j, \delta_{i j}=0$ for $i \neq j$ ). In statistically steady-state conditions, the equilibrium between the imposed pressure gradient and the sum $\tau_{s, d}^{u p}+\tau_{s, d}^{\text {down }}$ of the streamwise components of the lower- and upper-wall stresses holds. Specifically, it is

$\Pi_{d}=\frac{\tau_{s, d}^{u p}+\tau_{s, d}^{\text {down }}}{2 \delta}=\frac{\tau_{s, d}}{\delta}$,

where $\tau_{s, d}=\left(\tau_{s, d}^{u p}+\tau_{s, d}^{\text {down }}\right) / 2$ is the average wall stress, which is calculated as

$\tau_{s, d}=\frac{1}{2 A} \int_{A}\left[\mu \frac{\partial u_{s, d}}{\partial n} \mathbf{t} \cdot \mathbf{s}-p_{d} \mathbf{n} \cdot \mathbf{s}\right] d A$

where $A$ is the sum of the upper and lower wall surfaces, $u_{s}$ is the tangential velocity component, $\mathbf{t}$ and $\mathbf{n}$ are the tangential and normal unit vectors to the wall surface element $d A$ and $\mathbf{s}$ is the streamwise direction vector. Since the equations are made non-dimensional with the friction velocity $u^{*}=\sqrt{\tau_{s, d} / \rho}$ and the half-channel width $\delta$, it is always $\Pi=1$. In the Eqs. (1) and (2), the symbol $(\cdot)$ denotes filtered quantities. In statistically steady-state conditions, the equilibrium between the imposed pressure gradient and the overall streamwise components of the lower- and upper-wall stresses holds (for details, see DNA10). The subgrid-scale (SGS) stress tensor $\tau_{i j}$ is modeled using the Dynamic Mixed Model (DMM) of Zang et al. (1993):

$\tau_{i j}=\left(\overline{\bar{u}}_{i} \bar{u}_{j}-\overline{\bar{u}}_{i} \overline{\bar{u}}_{j}\right)-2 C \bar{\Delta}^{2}|\bar{S}| \bar{S}_{i j}$

where $\overline{\bar{u}}_{i} \bar{u}_{j}-\overline{\bar{u}}_{i} \overline{\bar{u}}_{j}$ is the modified Leonard term, $C$ is the dynamic Smagorinsky closure coefficient, $\bar{\Delta}$ is the filter width (with $\Delta$ the cube root of the cell volumes), $\bar{S}_{i j}$ is the resolved strain-rate tensor

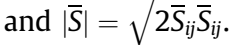

The momentum and continuity Eqs. (1) and (2) are resolved using the finite-volume numerical code PANORMUS (PArallel Numerical Open-souRce Model for Unsteady flow Simulations) (Napoli, 2011), which is second-order accurate both in time and space. The numerical model uses the explicit Adams-Bashforth method for the time advancement of the solution, while the fractional-step technique is used to overcome the pressure-velocity decoupling, in conjunction with the multigrid accelerator V-cycle (further details on the numerical procedure can be found in Lipari and Napoli (2008)). The numerical model was extensively and favorably validated over a wide range of cases (Cioffi et al., 2005, Jozsa et al., 2007, De Marchis and Napoli, 2008, De Marchis et al., 2010,2012 ). A discussion on the performed validation tests in turbulent channel flows is reported in Napoli et al. (2008).

The numerical simulations are performed using curvilinear structured boundary-fitted grids. Periodic boundary conditions are imposed in both the streamwise and spanwise directions, while the no-slip condition is enforced at the rough walls.

\subsection{Rough geometry and numerical parameters}

Two irregular rough surfaces are considered. The first one, as reported in DNA10, is obtained through the superimposition on a flat plane of sinusoidal functions with random amplitudes, as:

$r\left(x_{1}\right)=\sum_{i=1}^{n_{1}} A_{i} \sin \left(\frac{2 i \pi x_{1}}{L_{x_{1}} / 2}\right)$

where $r\left(x_{1}\right)$ is the wall distance from the flat reference surface, $L_{x_{1}}$ is the channel length, $n_{1}$ is the number of sinusoidal functions, $A_{i}$ and $L_{x_{1}} / 2 i$ are the amplitude and the wave-length of the $i$-th function in the streamwise direction, respectively. The roughness in this test case is two-dimensional, since the element heights depend on the $x_{1}$ coordinate only.

On the other hand, the second irregular rough surface is typically $3 \mathrm{D}$ and is obtained through the product of the superimposition of sinusoidal functions in the streamwise and spanwise directions, as: 


$$
\begin{array}{r}
r\left(x_{1}, x_{2}\right)=\sum_{i=1}^{n_{1}} A_{i} \sin \left(\frac{2 i \pi x_{1}}{L_{x_{1}} / 2}\right) . \\
\sum_{j=1}^{n_{2}} B_{j} \sin \left(\frac{2 j \pi x_{2}}{L_{x_{2}} / 2}\right),
\end{array}
$$

where $r\left(x_{1}, x_{2}\right)$ is the wall distance from the flat reference surface, $L_{x_{2}}$ is the channel width, $n_{2}$ is the number of sinusoidal functions in the spanwise direction, $B_{j}$ and $L_{x_{2}} / 2 j$ are the random amplitude and wave-length of the $j$ th function in the spanwise direction, respectively.

The amplitudes $A_{i}$ and $B_{j}$ with $i=j=1$ are set equal to 1 , while those of the higher wave-number functions are randomly generated in the range [0-1]. For both rough surfaces the sum of the sinusoidal functions is scaled in order to obtain the same values of the non-dimensional averaged absolute deviation $\bar{r}\left(x_{1}\right)$ and $\bar{r}\left(x_{1}, x_{2}\right)$. which can be calculated as:

$\bar{r}\left(x_{1}\right)=\frac{1}{\delta} \frac{1}{L_{x_{1}}} \int_{L_{x_{1}}}\left|r\left(x_{1}\right)\right| d x_{1}$

and

$\bar{r}\left(x_{1}, x_{2}\right)=\frac{1}{\delta} \frac{1}{L_{x_{1}} L_{x_{2}}} \int_{L_{x_{1}} L_{x_{2}}}\left|r\left(x_{1}, x_{2}\right)\right| d x_{1} d x_{2}$

In the present study, the $2 \mathrm{D}$ irregular rough surface is the case $C_{6}$ analyzed in DNA10, having an absolute non-dimensional deviation $\bar{r}\left(x_{1}\right)=0.05$. As demonstrated in DNA10, at the steady-state the fully rough regime is achieved. As discussed above, the same absolute deviation is imposed to the $3 \mathrm{D}$ rough wall. A similar procedure to generate rough surfaces was employed by Bhaganagar and Hsu (2009), who studied the effects of 2D and 3D ripples.

In Fig. 1a 3D representation of the rough walls is shown for both the $2 \mathrm{D}$ and $3 \mathrm{D}$ test cases. As can be seen, both upper and lower sur- faces are roughened through the superimposition of the sinusoidal functions. Since the amplitudes of the sinusoidal functions are generated randomly, the upper and lower surfaces are not identical. As in DNA10, the numerical simulations for the 2D and 3D irregular rough surfaces are carried out at $\operatorname{Re}_{\tau}=u^{*} \delta / v=395$. The choice to maintain a relatively low Reynolds number, in comparison with recent researches were higher values were analyzed (Scotti, 2006), is due to the increase in the computational costs caused by the irregularity of the wall in both directions, requiring a very high grid resolution in both the streamwise and spanwise directions. In DNA10 (2D rough case), in order to resolve the turbulence structures disrupted by the roughness elements, a relatively fine grid was used made of $256 \times 64 \times 64$ cells in the streamwise, spanwise and wall-normal directions, respectively. The domain length was $4 \pi$ and $\pi$ in the streamwise and spanwise directions, with grid points uniformly distributed in both the directions, with cell sizes $\Delta x_{1}^{+}=\Delta x_{2}^{+}=19$ wall units. The same grid resolution was used for the smooth-wall case. In the simulations conducted over the three-dimensional irregular rough walls, the same domain dimension has been considered but the grid has been further refined in the spanwise direction, in order to better reproduce the irregularity introduced by the superimposition of sinusoidal functions in that direction too. Thus, the domain has been subdivided into $256 \times 128 \times 64$ cells in the streamwise, spanwise and wall-normal directions, respectively, resulting in the spanwise grid spacing of $\Delta x_{2}^{+}=9.5$ wall units. In both cases, in the vertical direction a hyperbolic function was used to obtain a non-uniform mesh spacing with a refinement close to the upper and lower walls. The first calculus point was placed at a distance lower than one wall unit from the rough surfaces, while the cell size increased toward the channel centerline, with a maximum grid spacing of about 30 wall units. In Table 1 the geometrical data of the roughness are summarized, while the data on the domain size and mesh resolution are

(a)

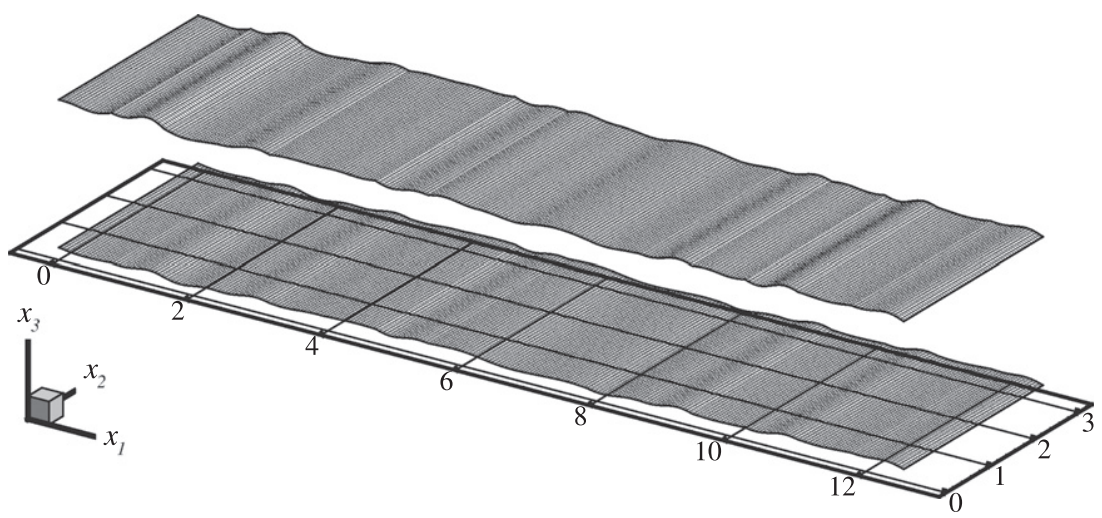

(b)

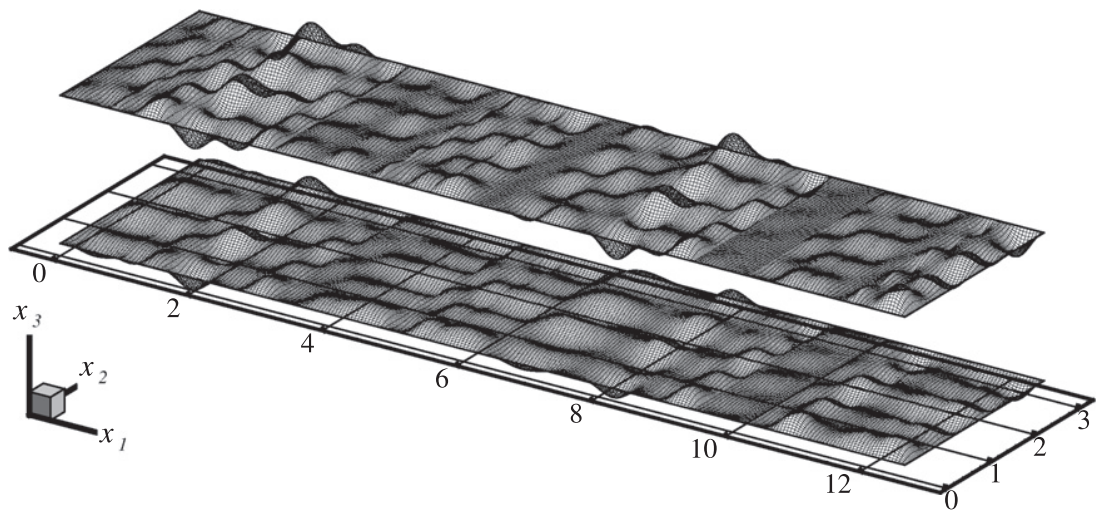

Fig. 1. 3D plot of the channel domains. (a) 2D irregular roughness; (b) 3D irregular roughness. 
Table 1

Geometrical parameter of the rough walls. $\bar{r}$ and $\bar{r}^{+}$: average absolute deviations of the heights of the rough walls made non-dimensional with the channel half-width $\delta$ and the wall units, respectively. Similarly, $h_{\text {rms }}$ and $h_{\text {rus }}^{+}$are the root mean squares of the wall oscillations, $k_{s}$ and $k_{s}^{+}$are the equivalent sand-grain roughness (obtained through the matching with the logarithmic wall-law), while $h_{\max }$ and $h_{\max }^{+}$are the highest roughness peaks. The values of the effective roughness ES, as defined in Napoli et al. (2008) is also reported.

\begin{tabular}{lllllllrrr}
\hline Case & $\bar{r}$ & $\bar{r}^{+}$ & $h_{r m s}$ & $h_{r m s}^{+}$ & $k_{s}$ & $k_{s}^{+} h_{\max }$ & $h_{\max }^{+} E S$ & \\
\hline 2D & 0.05 & 19.75 & 0.06 & 23.70 & 0.20 & 79.0 & 0.14 & 55.30 & 0.206 \\
3D & 0.05 & 19.75 & 0.07 & 27.70 & 0.36 & 142.2 & 0.37 & 146.15 & 0.239 \\
\hline
\end{tabular}

reported in Table 2 . The simulations have been carried out until the statistical time convergence was achieved, as demonstrated by the linear pattern of the total shear stress. In order to verify that the domain is long enough to reproduce the turbulence structures, two-point correlations have been analyzed both in the streamwise and spanwise directions, which are plotted at $x_{3}^{+}=15$ in the Figs. 2 and 3. In both directions all the correlations fall-off to null values at a distance lower than half domain. This result ensures that the domain size and resolution are sufficient to correctly resolve the turbulence structures. In Fig. 3a and $\mathrm{c}$ the profiles present a somewhat non-smooth pattern with some cusps within the first 100 wall units. This is due to the resolution of the grid (19 wall units in the spanwise direction) and to the rapid profile changes, assuming negative peaks at distances of some tenths of wall units. Nevertheless, it is worthwhile pointing out that the spanwise grid resolution is coherent with the observation of Piomelli and Balaras (2002), who suggested to use the grid spacing of 20 wall units in the spanwise direction in resolved LESs.

\section{Results and discussion}

\subsection{Mean velocity profiles}

One of the main effect of the wall roughness on the turbulent flows is the reduction of the streamwise velocity with respect to smooth-wall conditions. In the log-region, at sufficient distance from the roughness elements, the streamwise velocity profile can be written as:

$\frac{U}{u^{*}}=\frac{1}{k} \ln \left(y^{+}\right)+C_{s}-\Delta U^{+}$

where $U$ is the time-averaged streamwise velocity, $y^{+}$is the nondimensional wall-normal distance, $k$ is the von Kármán constant, $C_{s}$ is the additive constant (equal to about 5.5 for channel flows) and $\Delta U^{+}$is the roughness function. The value of the additive constant $C_{s}=5.5$ is used in Eq. (9), coherently with some literature findings (see among others, Bhaganagar et al. (2006), Orlandi et al. (2006), Bhaganagar and Hsu (2009)), which was obtained through the comparison of the smooth-wall numerical results with the logarithmic profiles $U^{+}=(1 / \kappa) \log \left(y^{+}\right)+C_{s}$ with different $C_{s}$ values. As discussed by Kim et al. (1987), higher value of $C_{s}$ with respect to the frequently achieved 5.0 value, can be considered, in turbulent channel flows, a low-Reynolds number effect. $\Delta U^{+}$is representative of the shift of the velocity profile in the vertical direction due to the increase of resistance induced by the wall irregularities with respect

Table 2

Domain size and mesh resolution.

\begin{tabular}{lllllllll}
\hline Case & $R e_{\tau}$ & $L_{x_{1}} / \delta$ & $L_{x_{2}} / \delta$ & $L_{x_{3}} / \delta$ & $\left(N_{x_{1}}, N_{x_{2}}, N_{x_{3}}\right)$ & $\Delta x_{1}^{+}$ & $\Delta x_{2}^{+}$ & $\Delta x_{3, \min }^{+}$ \\
\hline Smooth & 395 & $2 \pi$ & $\pi$ & 2 & $128,64,64$ & 19 & 19 & 1.6 \\
2D & 395 & $4 \pi$ & $\pi$ & 2 & $256,64,64$ & 19 & 19 & 1.6 \\
3D & 395 & $4 \pi$ & $\pi$ & 2 & $256,128,64$ & 19 & 9.5 & 1.6 \\
\hline
\end{tabular}

to smooth wall. The roughness function can be expressed as a function of the sand-grain roughness $k_{s}^{+}$, as suggested by Hama (1954):

$\Delta U^{+}=\frac{1}{k} \ln \left(k_{s}^{+}\right)+C_{s}-B$

with $B$ a constant equal to 8.5 .

The non-dimensional numerical mean velocity profiles for twoand three-dimensional roughness are shown in Fig. 4. In the figure the non-dimensional wall distance $y^{+}$is measured from the flat reference plane to which the sinusoidal functions are superimposed to generate the wall roughness (see Fig. 5). As discussed by Jackson (1981), $y^{+}$should be measured from the plane at which the total drag acts, frequently referred as "zero-plane displacement" and obtained through a procedure based on the theorem of the center of pressure. Nevertheless, when the position of this plane is calculated according to the definition and the procedure of Jackson (1981), it has been demonstrated that the best log-law fit requires a value of the Kármán's coefficient $k$ significantly lower than the classical value of 0.41 (Leonardi and Castro, 2010). Thus, several authors (see Cheng and Castro (2002), Flack and Schultz (2010) and literature therein reported) obtain the zero-plane displacement by optimizing the fit of the spatially averaged mean velocity to the log-linear profile, assuming $k=0.41$ and using the appropriate $u^{*}$ given by the calculated wall stress. Here, the same optimizing procedure is used, resulting in the identification of the zeroplane with the flat reference plane indicated in Fig. 5.

In order to quantify the overall effect of the wall irregularities, the velocities are averaged in time and in the $x_{1} x_{2}$-planes. Moreover, due to the small differences between the lower and the upper walls, the statistics are symmetrically averaged with respect to the mid-plane of the channel. In the following, the symbol <.> indicates variables averaged in time and in $x_{1} x_{2}$-planes, while the symbol \langle\rangle$_{t}$ will be used to indicate averaging in time only. In Fig. 4 the analytical profiles of equations $U^{+}=(1 / \kappa) \log \left(y^{+} / k_{s}^{+}\right)+8.5$ with $k_{s}^{+}=70$ (dividing the transitionally from the fully rough regime) is plotted too. The figure shows that in both numerical test cases the fully rough regime is achieved. Despite the two-dimensional and three-dimensional rough walls are characterized by the same value of $\bar{r}^{+}(\approx 20)$, the roughness function obtained with the 3D roughness is significantly higher than in the 2D case $\left(\Delta U^{+}=9.1\right.$ for the $3 \mathrm{D}$ case and $\Delta U^{+}=7.6$ for the $2 \mathrm{D}$ one). This indicates that the effects of 3D roughness on the mean velocities are higher than those of $2 \mathrm{D}$ roughness, at least for the irregular pattern here analyzed. This result is in contrast with the recent findings of Lee et al. (2011) who, comparing the effect of a 3D cube-roughened wall on the turbulent flow with those of a 2D rod-roughened wall, obtained an higher downward shift of the velocity profile with the $2 \mathrm{D}$ rough elements. The difference can be probably due to the specific configuration of the 3D rough wall considered by Lee et al. (2011), which was generated through cube blocks periodically arranged over a flat plate. This cube arrangement, in fact, creates channels between the rough-cubes, thus reducing the flow impingement. On the other hand, the results here shown are consistent with those obtained by Bhaganagar and Hsu (2009) in their analysis of 2D and 3D ripples. The agreement of our results with those obtained over wavy roughness and the misalignment with those over walls roughened through the periodical arrangement of regular sharp-edge elements can be also attributed to the shape of the roughness elements, producing different effects on the turbulent flow. This result is coherent with the analysis carried out by Schultz and Flack (2009) who identified, on the basis of the effective slope (ES), the waviness and the roughness flow regimes, with the latter occurring for the highest values of ES typical of sharp-edge rough geometries.

The different values of the roughness function obtained with the two rough surfaces, characterized by the same value of the 

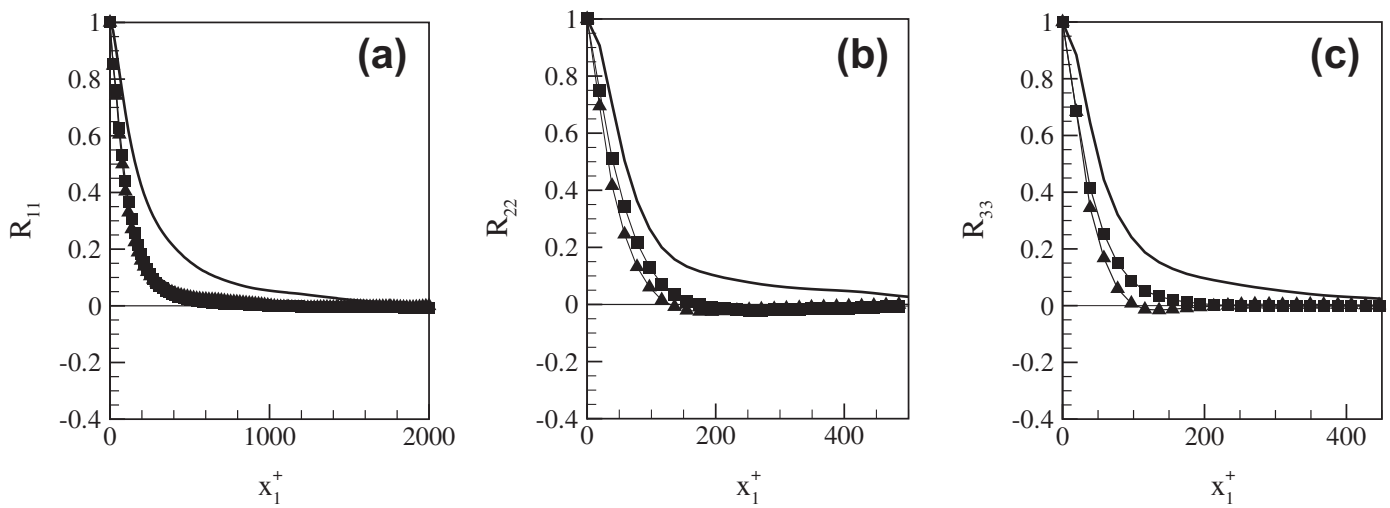

Fig. 2. Streamwise two-point correlation at $x_{3}^{+}=15$ : -, smooth wall; $-\mathbf{\Lambda}-, 2 \mathrm{D}$ roughness; component; (c) velocity wall-normal component.
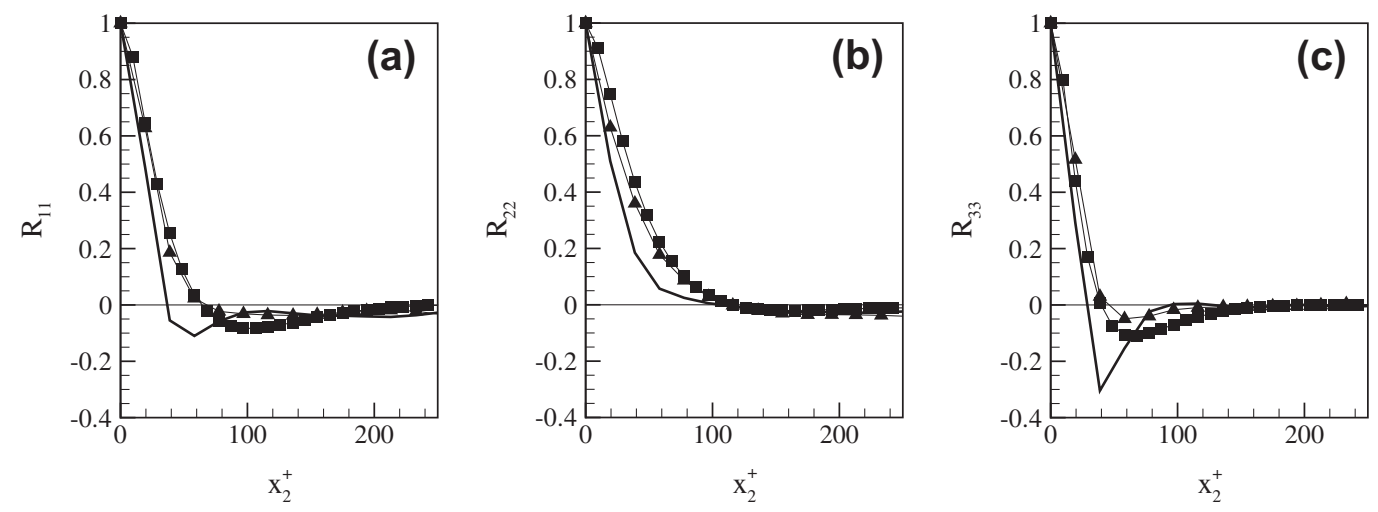

Fig. 3. Spanwise two-point correlation at $x_{3}^{+}=15$ : -, smooth wall; $-\mathbf{\Delta}-$, 2D roughness; component; (c) velocity wall-normal component.

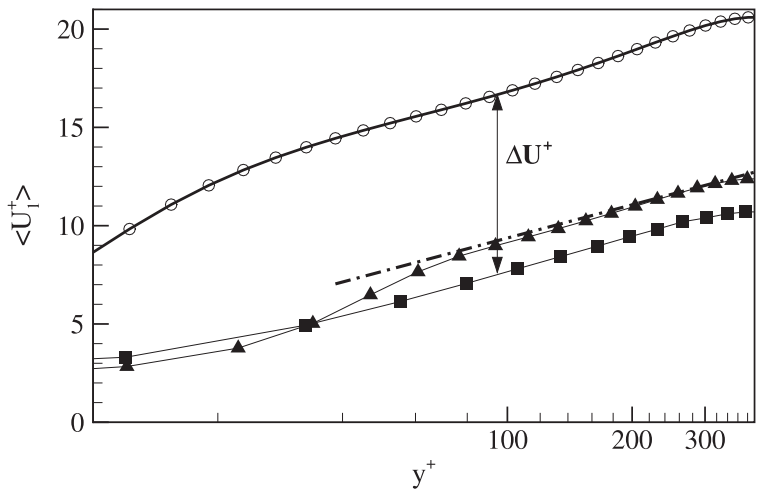

Fig. 4. Mean velocity profiles for the analyzed cases: -o-, 3D hydraulically smooth

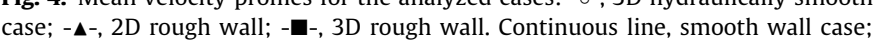
dot-dash line, limit of the fully rough wall: $\left\langle U_{1}^{+}\right\rangle=(1 / \kappa) \log \left(y^{+} / k_{s}^{+}\right)+8.5$ with $k_{s}^{+}=70$.

mean amplitude $\bar{r}^{+}$of the roughness, show that the parameter $\bar{r}^{+}$ cannot be considered a suitable geometric index to fully characterize the irregular roughness. As demonstrated by Napoli et al. (2008) and Schultz and Flack (2009) (and literature therein), the key geometric parameter representative of the wall roughness should take into account the density of the roughness elements too. Specifically, Napoli et al. (2008) introduced the Effective Slope $(E S)$ to characterize the behavior of irregular rough walls, which was later verified by Schultz and Flack (2009) over regular threedimensional roughness. The two geometries here considered share

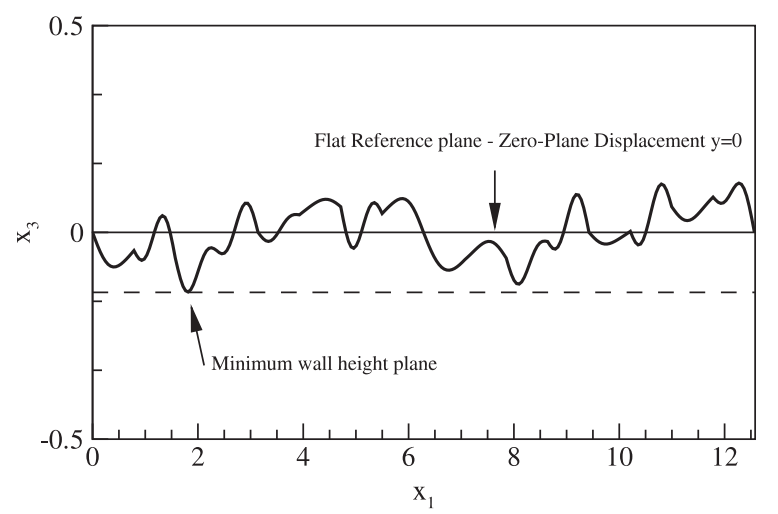

Fig. 5. Sketch of the irregular rough wall, with indication of the flat reference plane and the displacement height $d$.

the same mean amplitude $r^{+}$but are characterized by slightly different values of ES. The value of ES for the three-dimensional rough wall, in fact, as reported in Table 1 , is equal to 0.239 , while the effective slope for the two-dimensional roughness is 0.206 . The higher value of ES for the 3D roughness thus can explain the increase of the roughness function since, as it is seen in Fig. 4, in the considered range of ES values the roughness function is quite sensitive to the changes in the effective slope (while for values larger than $\simeq 0.4$ no clear dependence of $\Delta U^{+}$on $E S$ can be identified). Nevertheless, since the difference between the considered values of ES is not very high (less than $20 \%$ ), the obtained result could 
be due to the larger height of the roughness elements in the 3D roughness case with respect to the $2 \mathrm{D}$ one (as reported in Table 1 , in fact, the 3D highest roughness peak is about three times larger than the $2 \mathrm{D}$ peak). In fact, as recently pointed out by Xie et al. (2008), the tallest elements can dominate the local turbulence, with effects on the spatially-averaged profiles too.

In Fig. 6 the values of the roughness function obtained in the numerical simulations of the turbulent flows over the irregular 2D and 3D rough walls are plotted against the effective slope. The results obtained by Napoli et al. (2008) and Schultz and Flack (2009), considering 2D and 3D rough geometries, respectively, are shown too. The point representative of the 3D irregular rough surface exhibits a good agreement with the other points, showing that, also for the irregular 3D roughness shapes, the effective slope is one of the geometric parameters able to represent the effect of a rough wall on the roughness function. In order to confirm this result, further simulations will be carried out in future researches.

The analysis of the velocity defect, plotted in Fig. 7, shows that the profiles obtained with the $2 \mathrm{D}$ and $3 \mathrm{D}$ rough surfaces are similar one each other and to the smooth-wall condition in the most of the outer layer, although in the range between 70 and 200 wall units some differences can be observed (with values over the 2D and $3 \mathrm{D}$ rough surfaces about $10 \%$ lower than over the smooth wall). In the near-wall region, on the other hand, the plot confirms that $3 \mathrm{D}$ roughness produces a more pronounced velocity reduction as compared to the $2 \mathrm{D}$ roughness. This result is in agreement with the findings of several authors for both 2D and 3D rough walls (Antonia and Krogstad, 2001, Ashrafian et al., 2004, Bhaganagar et al., 2006, Lee et al., 2011) and represents the first indicator of the outer layer independence on the surface roughness. In order to further investigate on this issue, the mean velocity profiles have been then scaled with the outer layer length scales. As suggested by Bakken et al. (2005), in a channel flow the only length scale for the coordinates in the outer layer is the channel half-height $\delta$. On the other hand, different velocity scales have been proposed in the literature. Zagarola and Smits (1998), and later several authors, suggested that a reasonable candidate for the outer velocity scale is the centerline streamwise velocity $\left\langle U_{c l}\right\rangle$, averaged in time and over the channel mid-plane. The velocity defect, scaled with the centerline velocity, is thus plotted against the distance $y / \delta$ in Fig. 8. The profiles obtained for the rough surfaces differ from the smooth wall one not only in the wall proximity, but in most of the outer region too. This result is not in contrast with the wall similarity and was expected in light of the downward shift of the mean velocity profile as seen in Fig. 4. Moreover, it is consistent with the findings of Brzek et al. (2007) in their experiments of turbulent flows over irregular three-dimensional rough surfaces and with those of Akinlade et al. (2004), who concluded that using

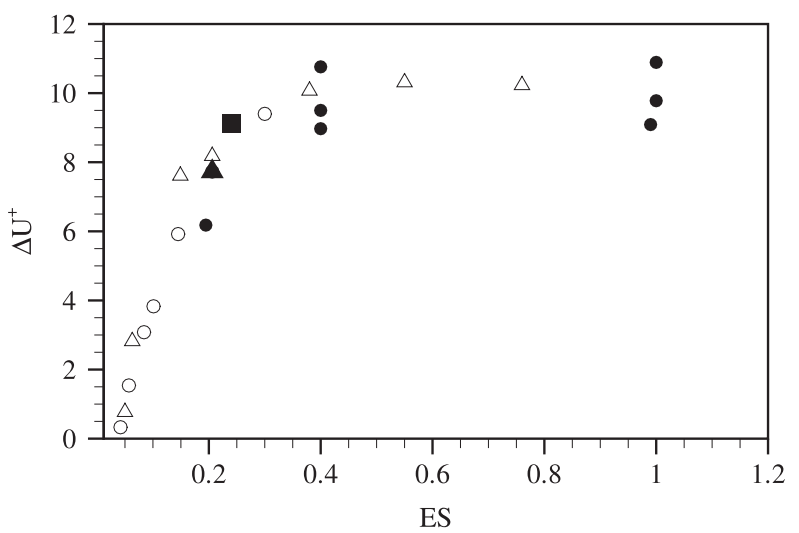

Fig. 6. $\Delta U^{+}$in function of the effective slope ES: $\mathbf{\Delta}, 2 \mathrm{D}$ roughness; $\mathbf{\square}, 3 \mathrm{D}$ roughness; $\Delta$, (Napoli et al., 2008); ॰, (De Marchis et al., 2010); •, (Schultz and Flack, 2009).

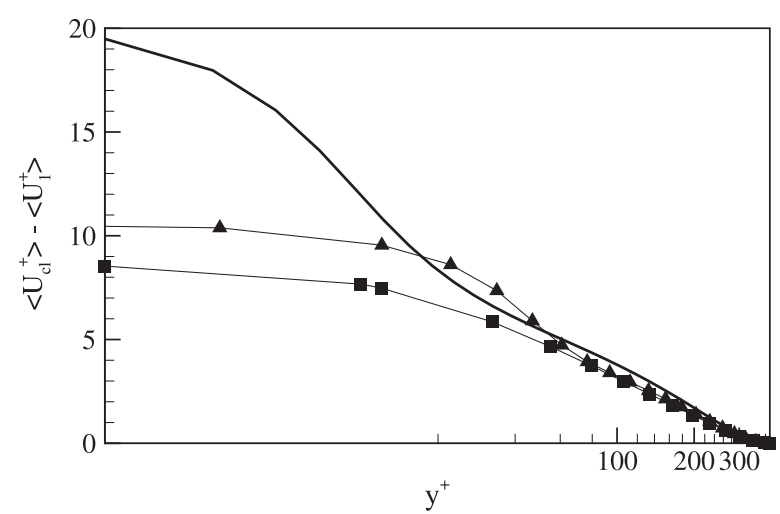

Fig. 7. Inner layer scaling of the mean velocity defect: continuous line, smooth wall case; -o-, numerical results in the hydraulically smooth case; $-\mathbf{\Delta}-$, numerical results with $2 \mathrm{D}$ roughness; - $\mathbf{-}-$; numerical results with 3D roughness.

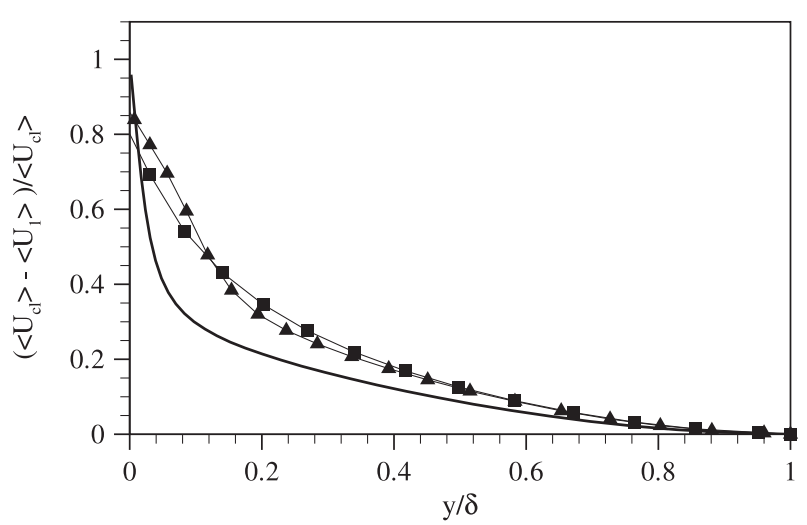

Fig. 8. Outer layer scaling of the mean velocity defect. Symbols as in Fig. 7.

the centerline velocity as a scale does not allow the velocity profiles for smooth and rough walls to collapse.

\subsection{Reynolds stress analysis}

In order to obtain further insights on the irregular 2D and 3D roughness effects, higher order statistics have been taken into account as well. Specifically, in this section an analysis is performed of the root mean squares (rms) of the velocity fluctuations, indicated as $u_{i, r m s}$. The contribution of the sub-grid scale terms, modeled with the Dynamic Mixed Model, is added to the resolved

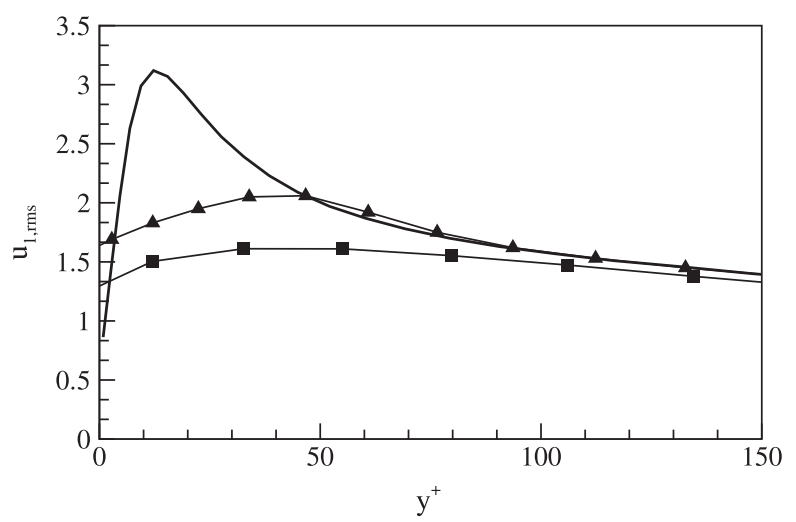

Fig. 9. Profiles of the streamwise component of the rms of the velocity fluctuations: -, smooth wall; - $-\mathbf{\Delta - ,}$ 2D roughness; - $\mathbf{-}-$, 3D roughness. 
values. In Fig. 9 the rms of the velocity fluctuation in the streamwise direction $u_{1, r m s}$, normalized by the friction velocity, is plotted in inner coordinates. The profiles obtained with $2 \mathrm{D}$ and $3 \mathrm{D}$ roughness, coherently with the recent findings of De Marchis et al. (2010) and Lee et al. (2011), almost collapse in the external region, while showing large differences in the inner layer. The peaks of the turbulent intensities, which are located close to the wall in the smooth wall case, disappear for both 2D and 3D configurations, that exhibit on the contrary almost flat profiles. This behavior is more evident for the $3 \mathrm{D}$ roughness than for the $2 \mathrm{D}$ one. Moreover, the deviation from the smooth profile for the 3D rough wall is clearly evident in a slightly larger region than for the $2 \mathrm{D}$ roughness. Specifically, the profile of the streamwise component of the rms of the velocity fluctuations for the 3D rough case is constantly shifted downward of about $5 \%$ with respect to the $2 \mathrm{D}$ case. This can be attributed to the highest values of the maximum wall peaks in the $3 \mathrm{D}$ case, that increase the interaction between the inner and outer layers.

The spanwise components of the rms of the velocity fluctuations $u_{2, r m s}$ obtained over the smooth wall and the two rough surfaces show even larger differences. Specifically, the 2D roughness produces an increase of the spanwise component in the near-wall region, while at distances greater than 20 wall units only small differences can be seen in the profiles. On the other hand, the 3D rough wall causes an increase of the spanwise component $u_{2, r m s}$ in a thin layer of about 10 wall units and a slight reduction in the region between 10 and 80 wall units, showing an almost flat profile. In the external layer the profiles for the smooth wall, the 2D and the 3D roughness collapse (see Fig. 10).

The differences between the $2 \mathrm{D}$ and the $3 \mathrm{D}$ roughness are quite lower when analyzing the wall-normal component $u_{3, r m s}$ (Fig. 11). In this case the deviation from the smooth-wall profile is quite evident up to about 100 wall units, although again some small differences can be seen in the whole outer layer.

The results discussed so far show that 3D irregularities cause higher deviations from the smooth wall than the ones observed with $2 \mathrm{D}$ roughness. The reason for this behavior can be due to the impingement of the flow on the three-dimensional peaks of the roughness, giving rise to a higher interaction between the streamwise, spanwise and wall-normal components of the fluctuations. Moreover, three-dimensional roughness increases the tendency toward isotropization with respect to two-dimensional roughness (De Marchis et al., 2010).

In Fig. 12 the profiles of the Reynolds shear stresses $\left\langle u_{1}^{\prime} u_{3}^{\prime}\right\rangle$ are plotted in viscous units for the two considered rough cases (where $u_{i}^{\prime}$ is the total turbulent fluctuation). In the figure the viscous stress $v \frac{\partial\left\langle U_{1}\right\rangle}{\partial x_{3}}$ and the pressure stress $\Delta P$ are plotted too for both the $2 \mathrm{D}$ and 3D roughness. Moreover, in order to obtain the expected steadystate linear pattern, the contribution of the dispersive stress (Finn-

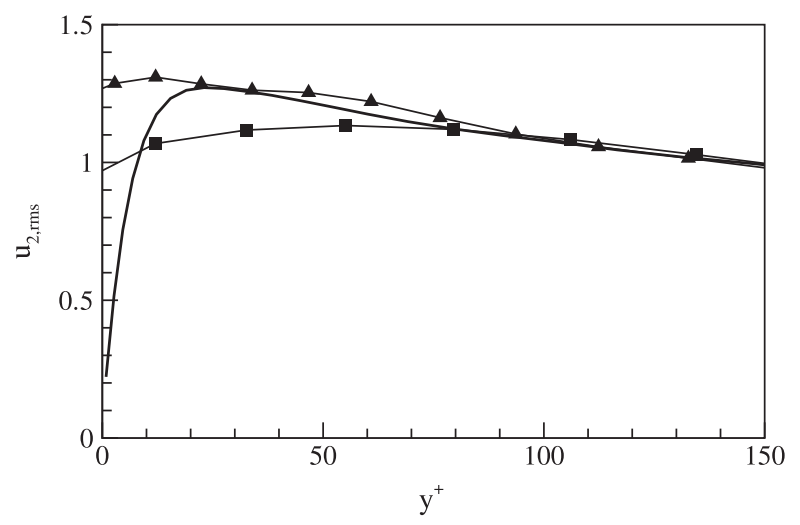

Fig. 10. Profiles of the spanwise component of the rms of the velocity fluctuations:

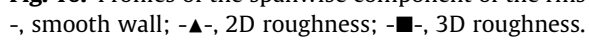

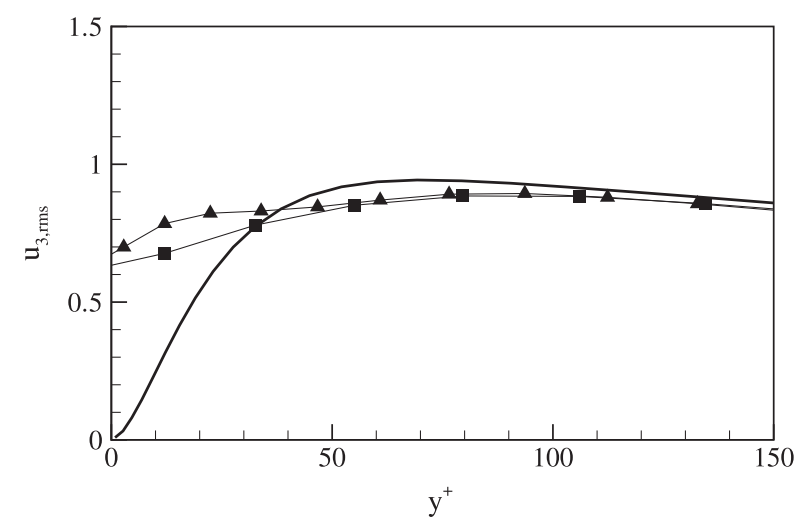

Fig. 11. Profiles of the wall-normal component of the rms of the velocity fluctuations: -, smooth wall; - $\mathbf{\Delta -}-, 2 \mathrm{D}$ roughness; -ш-, 3D roughness.
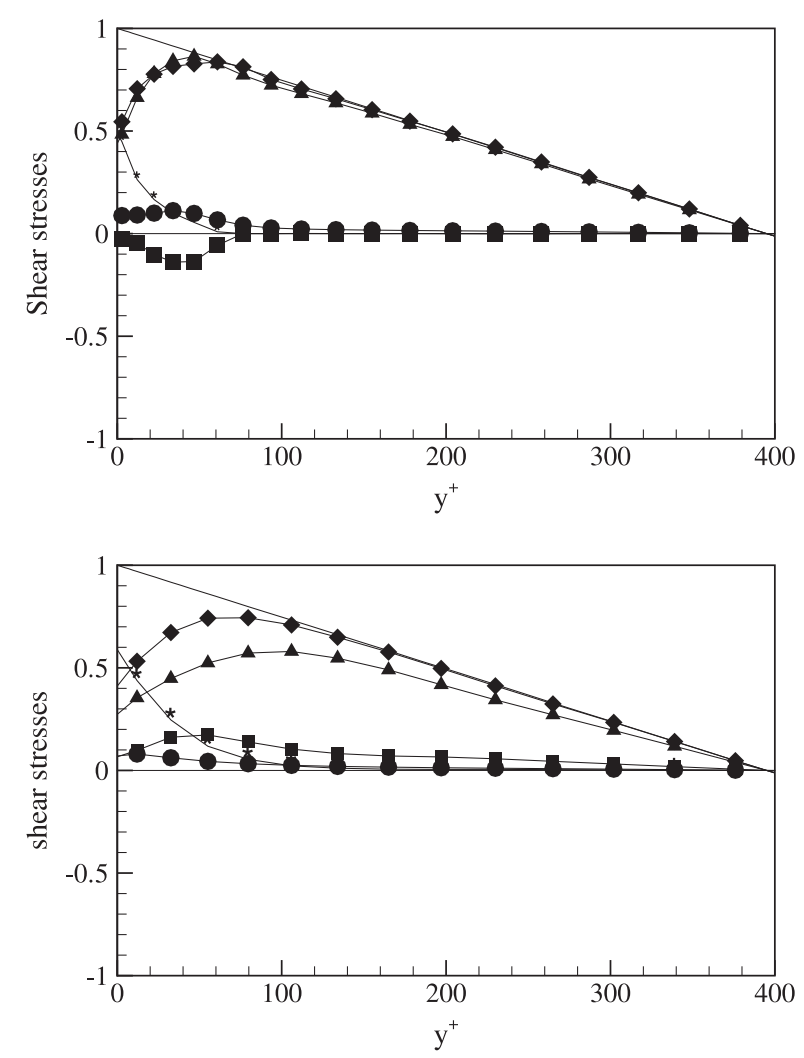

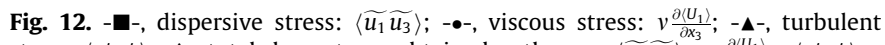
stress: $\left\langle u_{1}^{\prime} u_{3}^{\prime}\right\rangle ;--$, total shear stress, obtained as the sum $\left\langle\widetilde{u_{1}} \widetilde{u_{3}}\right\rangle+v \frac{\partial\left\langle u_{1}\right\rangle}{\partial x_{3}}+\left\langle u_{1}^{\prime} u_{3}^{\prime}\right\rangle$; $\checkmark ;-*-$, pressure stress: $\Delta \mathrm{P}$; the straight line is the sum of the total shear stress and $\Delta \mathrm{P}$. Top: 2D roughness; bottom: 3D roughness. All the values are made nondimensional with the squared friction velocity $u^{*^{2}}$.

igan, 2000; Coceal et al., 2006), obtained as $\left\langle\widetilde{u_{1}} \widetilde{u_{3}}\right\rangle$, with $\widetilde{u}_{i}=\left\langle u_{i}\right\rangle_{t}-\left\langle u_{i}\right\rangle$, must be added. These terms, accounting for the non-homogeneity in the streamwise and spanwise directions, are very different in the $2 \mathrm{D}$ and $3 \mathrm{D}$ cases. The dispersive stress contribution, in fact, in the 2D case is limited to the region up to about 80 wall units, while in the $3 \mathrm{D}$ case is non-negligible for the whole channel height, thus indicating that some non-homogeneity exists in the $x_{1} x_{2}$-planes even in the outer region due to the 3D roughness. Moreover, the dispersive stress is negative in the 2D rough wall, while its sign is positive in the $3 \mathrm{D}$ case.

The straight line obtained by summing up the contributions of the viscous, turbulent and dispersive terms to the pressure force ensures that the statistical steady-state is achieved in the numeri- 
cal simulations and that the time period chosen to calculate the statistics (equal to 40 non-dimensional time units $t u^{*} / \delta$ for the $2 \mathrm{D}$ roughness and to $80 \mathrm{tu} / \delta$ for the $3 \mathrm{D}$ roughness) is sufficient to analyse the turbulent flow.

As suggested by several authors (see, among others, Antonia and Krogstad (2001); Leonardi et al. (2006); De Marchis et al. (2010)), the ratio between the Reynolds stresses is a suitable indicator to check the turbulence isotropy. It is well known, in fact, that the roughness induces a tendency towards turbulence isotropy close to the walls. To this aim, the symmetric and traceless Reynolds stress anisotropy tensor can be used, defined as:

$b_{i j}=\frac{\left\langle u_{i}^{\prime} u_{j}^{\prime}\right\rangle}{q^{2}}-\frac{1}{3} \delta_{i j}$

where $q^{2}=\left\langle u_{k}^{\prime} u_{k}^{\prime}\right\rangle$ is twice the mean turbulent kinetic energy and $\delta_{i j}$ is the Kronecker function. The tensor $b_{i j}$ is bounded by the values $-1 / 3$ and $2 / 3$ and reaches the null value for isotropic turbulence. The components $b_{11}, b_{22}, b_{33}$ and $b_{13}$ are plotted in Fig. 13 , that clearly shows large differences between smooth and rough walls in the near-wall region. Nevertheless, coherently with the observation of the rms of the velocity fluctuations, in the outer layer too some deviations (in the order of about $10 \%$ ) can be seen with respect to the smooth-wall case. The plots confirm that 3D roughness is more effective than the $2 \mathrm{D}$ one in reducing the turbulence anisotropy. The deviations of the $b_{i i}$ terms from the smooth wall case, in fact, are larger for the 3D roughness both in the inner and external regions, leading to nearly flat profiles. A similar finding was achieved by Antonia and Krogstad (2001) in their comparison of rod and wire-mesh rough surfaces.

The larger tendency toward isotropy induced by the 3D roughness is more clearly evident in Fig. 14, where the triangle of the Anisotropy Invariant Map (AIM) (Lumley, 1978; Smalley et al., 2002) is shown. In the figure the comparison between the AIMs obtained with the smooth wall, the $2 \mathrm{D}$ and $3 \mathrm{D}$ rough surfaces is shown. Coherently with the DNS data of Kim et al. (1987), at the distance $y^{+}=7$ from the flat plane the smooth-wall map approaches the right vertex of the triangle, which is representative of one-component state of turbulence. At larger distances from the wall the profile moves parallel to the right boundary of the triangle, characteristic of a rod-like (or cigar-shaped, Choi and Lumley (2001)) tur-

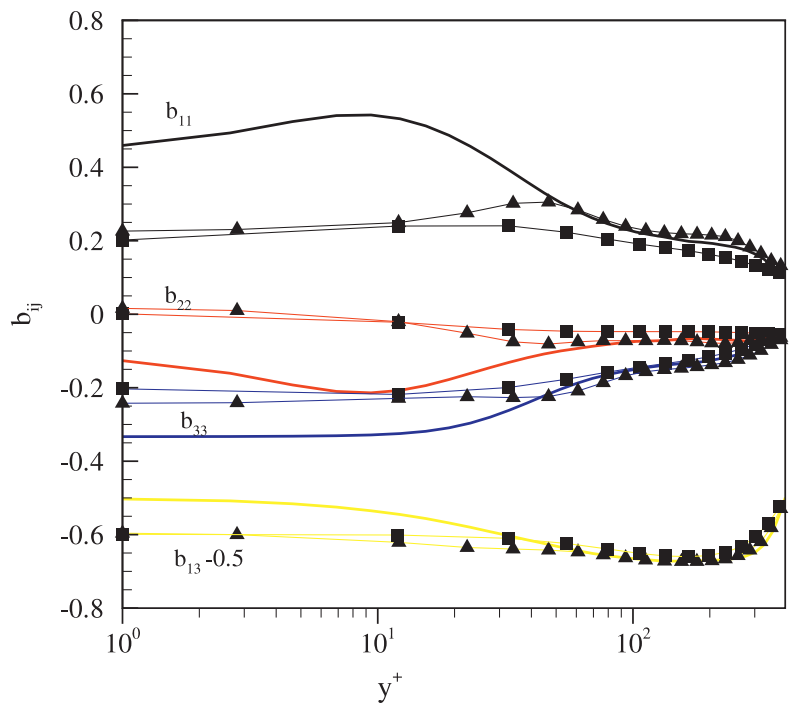

Fig. 13. Profiles of the components of the Reynolds stresses anisotropy tensor: Solid-line, smooth wall; - $\mathbf{\Delta -}-, 2 \mathrm{D}$ roughness; - $\mathbf{-}-$, 3D roughness. In order to improve the clarity, $b_{11}$ terms are drawn in black, $b_{22}$ in red, $b_{33}$ in blue and $b_{13}$ in yellow. (For interpretation of the references to color in this figure legend, the reader is referred to the web version of this article.)

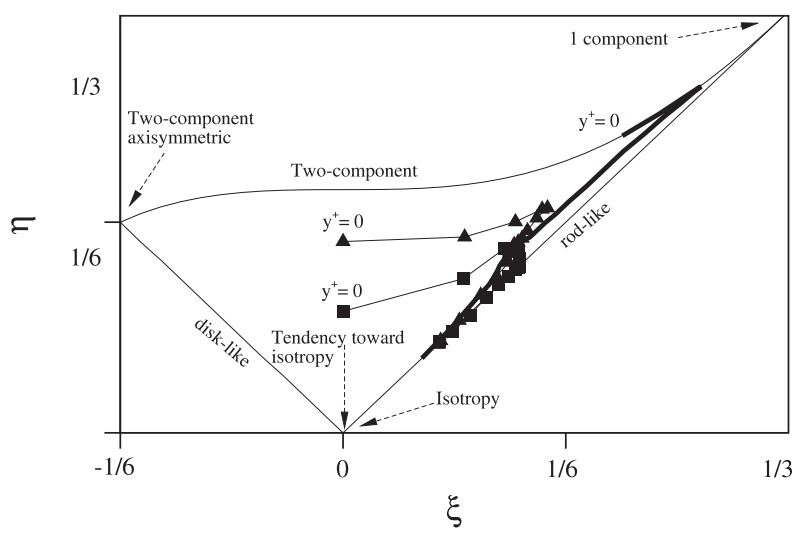

Fig. 14. Anisotropy Invariant Map: Bold solid-line, smooth wall; -,-- 2 D roughness; - - , 3D roughness. $\eta=b_{i j} b_{j i}$ and $\xi=b_{i j} b_{j k} b_{k i}$ are the second and third invariants of the Reynolds stress anisotropy tensor.

bulent state having one diagonal component much greater than the others. By moving further away from the wall, the profile is shifted toward the right line, in a step-like manner. Fig. 14 clearly highlights the drastic differences between the characteristics of the turbulence over smooth and rough surfaces, especially near the walls. Specifically, it can be observed that close to the wall the 3D roughness increases the tendency toward isotropy, identified by the map bottom cusp, with respect to the $2 \mathrm{D}$ roughness. Moreover, moving toward the center of the domain, the map obtained with the considered 2D roughness shows a behavior similar to the smooth wall one, with a step-like shift toward the rod-like line. On the other hand, when the three-dimensional roughness is considered, the step-like shift disappears. This result shows some similarity with the observation of Smalley et al. (2002), who noticed that, in turbulent flows over walls roughened with regularly arranged two-dimensional bars, a shift similar to the smooth case is identified, which is not present over 3D rough surfaces given by wire meshes or perforated plates. Finally, close to the centerline of the channel, a tendency to isotropy is clearly seen irrespective of the wall geometries, thus confirming that also for three-dimensional irregular rough geometries the wall similarity is achieved.

\section{Summary and concluding remarks}

Large Eddy Simulations of fully developed turbulent channel flows have been carried out to analyse the main differences between two- and three-dimensional irregular roughness. The roughness was obtained through the superimposition on the flat walls of random amplitude sinusoidal functions applied in the streamwise and spanwise directions (3D case) and in the streamwise direction only (2D case). The two irregular surfaces were built in such a way as to obtain the same mean roughness height $\bar{r}^{+} \approx 20$. Both the upper and the lower walls were roughened and the simulations were performed at the same relatively low friction Reynolds number $R e_{\tau}=395$.

An analysis of the first- and second-order statistics was performed to compare the smooth-wall and the two rough-wall behaviors, taking into account the recent findings on the turbulent flows over regular rough surfaces.

The analysis of the mean velocity profiles showed that the roughness function obtained with the 3D roughness is about $20 \%$ higher than in the 2D roughness case, although the two surfaces share the same mean roughness height. Thus the effect of threedimensional irregularities is higher than that of two-dimensional roughness and the mean amplitude of the roughness height cannot be considered a suitable parameter fully representative of the 
roughness effect, at least for irregular geometries. Other geometrical parameters such as the equivalent sand grain roughness $k_{s}$ or the effective slope ES seem to be more effective in representing the roughness effects on the velocity profile and on the related roughness function. The higher value of the roughness function achieved in the 3D configuration with respect to the 2D one, in fact, is coherent with the higher value of the effective slope, with results in very good agreement with the curve $\Delta U^{+}$vs ES built with the data of Napoli et al. (2008) and Schultz and Flack (2009). The velocity defect plot showed that the considered irregular roughness, while producing drastic deviations from the smooth-wall behavior in the near-wall region, causes only little differences in the outer layer, thus giving support to the Townends wall similarity hypothesis. The analysis of the rms's of the velocity fluctuations, as well as of the shear stresses, too, showed that the largest deviations from the smooth-wall conditions, both for the $2 \mathrm{D}$ and the 3D roughness, are confined to the region close to the wall. Nevertheless, the streamwise and wall-normal components of the turbulence intensity clearly show that, for the rough geometries here considered, some effects of the wall roughness can be observed in the outer layer too, where a reduction of about $10 \%$ with respect to the smooth wall is obtained.

The rms's of the velocity fluctuations, as well as the shear stresses, in fact, show that the deviations from the smooth wall conditions, both for the $2 \mathrm{D}$ and the $3 \mathrm{D}$ roughness, are confined in a region close to the wall and that in the external region an overlap of all the profiles is obtained. Coherently with the results of the analysis of the first-order statistics, the observation of the turbulence intensity confirmed that the 3D roughness induces higher effects than the 2D one. The tendency toward isotropy due to the roughness is enhanced too by $3 \mathrm{D}$ irregularities, as demonstrated by the Anisotropy Invariant Map obtained with the results of our simulations.

In conclusion, the present analysis pointed out that the considered 3D irregular rough surface produces higher effects on the turbulent flow than those observed over the 2D irregular roughness. Moreover, due to the specific random nature of the considered roughness, only geometric parameters able to take into account the shape, the distribution and the height of the irregularities can be considered representative of the specific wall configuration. This is typical of the "waviness regime", occurring for $E S<\sim 0.35$ as suggested by Schultz and Flack (2009), where the roughness function does not scale with the roughness Reynolds number, while the opposite occurs in the "roughness regime" (where the form drag on the roughness elements is much larger than the frictional drag).

The results obtained with the numerical simulations of the turbulent flows over irregular roughness share some features with most of the literature findings obtained over rough wall arranged in a regular patterns. Further efforts are needed to investigate on the specific differences between three-dimensional regular and irregular roughness. Ongoing numerical experiments attempt to address this challenge.

\section{References}

Acharya, M., Bornstein, J., Escudier, M.P., 1986. Turbulent boundary layers on rough surfaces. Exp. Fluids 4, 33-47.

Akinlade, O.G., Bergstrom, D.J., Tachie, M.F., Castillo, L., 2004. Outer flow scaling of smooth and rough wall turbulent boundary layers. Exp. Fluids 37, 604-612.

Antonia, R.A., Krogstad, P.A., 2001. Turbulent structure in boundary layers over different types of surface roughness. Fluid Dynam. Res. 28, 139-157.

Ashrafian, A., Anderson, H.I., Manhart, M., 2004. DNS of turbulent flow in a rodroughness channel. Int. J. Heat Fluid Flow 25, 373-383.

Bakken, O.M., Krogstad, P.A., Ashrafian, A., Andersonn, H.I., 2005. Reynolds number effects in the outer layer of the turbulent flow in channel with rough walls. Phys. Fluids 17 (065101).

Bhaganagar, K., Kim, J., Coleman, G., 2006. Effect of roughness on wall-bounded turbulence. Flow Turbul. Combust. 72, 463-492.
Bhaganagar, K., Hsu, T.J., 2009. Direct numerical simulations of flow over twodimensional and three-dimensional ripples and implication to sediment transport: steady flow. Coast. Eng. 38, 320-331.

Birch, D.M., Morrison, J.F., 2011. Similarity of the streamwise velocity component in very-rough-wall channel flow. J. Fluid Mech. 668, 174-201.

Bons, J.P., Taylor, R.P., McClain, S.T., Rivir, R., 2001. The many manifestations of turbine surface roughness. J. Turbomach. 123 (4), 739-748.

Bons, J.P., 2002. $\mathrm{S}_{\mathrm{t}}$ and $\mathrm{c}_{\mathrm{f}}$ augmentation for real turbine roughness with elevated freestream turbulence. J. Turbomach. 124 (4), 632-645.

Brzek, B., Cal, R.B., Johansson, G., Castillo, L., 2007. Inner and outer scalings in rough surface zero pressure gradient turbulent boundary layers. Phys. Fluids 19, 065101.

Cheng, H., Castro, I.P., 2002. Near wall flow over urban-like roughness. Bound.-Layer Meteorol. 104, 229-259.

Choi, K.S., Lumley, J.L., 2001. The return to isotropy of homogeneous turbulence. J. Fluid Mech. 436, 59-84.

Cioffi, F., Gallerano, F., Napoli, E., 2005. Three-dimensional numerical simulation of wind driven flows in closed channels and basins. J. Hydraul. Res. 43 (3), 290 301.

Coceal, O., Thomas, T.G., Castro, I.P., Belcher, S.E., 2006. Mean flow and turbulence statistics over groups of urban-like cubical obstacles. Bound.-Layer Meteorol. $121,491-519$.

Colebrook, C.F., 1939. Turbulent flow in pipes with particular reference to the transition region between the smooth- and rough-pipe laws. J. Inst. Civil Eng. $11,133-156$.

Connelly, J.S., Schultz, M.P., Flack, K.A., 2006. Velocity-defect scaling for turbulent boundary layers with a range of relative roughness. Exp. Fluids 40, 188-195.

De Marchis, M., Napoli, E., 2008. The effect of geometrical parameters on the discharge capacity of meandering compound channels. Adv. Water Res. 31, 1662-1673.

De Marchis, M., Napoli, E., Armenio, V., 2010. Turbulence structures over irregular rough surfaces. J. Turbul. 11 (3), 1-32.

De Marchis, M., Ciraolo, G., Nasello, C., Napoli, E., 2012. Wind- and tide-induced currents in the Stagnone lagoon (Sicily). Environ. Fluid Mech. 12 (1), 81100.

Djenidi, L., Elevarasan, R., Antonia, R.A., 1999. The turbulent boundary layer over transverse square cavities. J. Fluid Mech. 395, 271-294.

Finnigan, J., 2000. Turbulence in plant canopies. Annu. Rev. Fluid Mech. 32, 519571.

Flack, K.A., Schultz, M.P., Shapiro, T.A., 2005. Experimental support for Townsend's Reynolds number similarity hypothesis on rough walls. Phys. Fluids 17 (035102-035102-9), 1-9.

Flack, K.A., Schultz, M.P., 2010. Review of hydraulic roughness scales in the fully rough regime. J. Fluids Eng. 132, 1-10.

Ikeda, T., Durbin, P., 2007. Direct simulations of a rough-wall channel flow. J. Fluid Mech. 561, 235-263.

Hama, F.R., 1954. Boundary layer characteristics for smooth and rough surfaces. Trans. Soc. Naval Arch. Marine Eng. 62, 333-358.

Jackson, P.S., 1981. On the displacement height in the logarithmic profiles. J. Fluid Mech. 111, 15-25.

Jimenez, J., 2004. Turbulent flows over rough walls. Annu. Rev. Fluid Mech. 36, 173 196.

Jozsa, J., Milici, B., Napoli, E., 2007. Numerical simulation of internal boundary-layer development and comparison with atmospheric data. Bound.-Layer Meteorol. 123 (1), 159-175.

Kim, J., Moin, P., Moser, R., 1987. Turbulence statistics in fully developed channel flow at low Reynolds number. J. Fluid Mech. 177, 133-166.

Krogstad, P.A., Antonia, R.A., 1999. Surface roughness effects in turbulent boundary layers. Exp. Fluids 27, 450-460.

Lee, J.H., Sung, H.J., Krogstad, P.A., 2011. Direct numerical simulation of the turbulent boundary layer over a cube-roughened wall. J. Fluid Mech. 669, 397431.

Leonardi, S., Orlandi, P., Smalley, R.J., Djenidi, L., Antonia, R.A., 2003. Direct numerical simulations of turbulent channel flow with transverse square bars on one wall. J. Fluid Mech. 491, 229-238.

Leonardi, S., Orlandi, P., Djenidi, L., Antonia, R.A., 2006. Guidelines for modeling a 2D rough wall channel flow. Flow Turbul. Combust. 72, 463-492.

Leonardi, S., Castro, I.P., 2010. Channel flow over large cube roughness: a direct numerical simulation study. J. Fluid Mech. 651, 519-539.

Lipari, G., Napoli, E., 2008. The impacts of the ale and hydrostatic-pressure approaches on the energy budget of unsteady free-surface flows. Comput Fluids 37 (6), 656-673.

Lumley, J.L., 1978. Computational modeling of turbulent flows. Adv. Appl. Mech. 18, 123-176.

Napoli, E., Armenio, V., De Marchis, M., 2008. The effect of the slope of irregularly distributed roughness elements on turbulent wall-bounded flows. J. Fluid Mech. $613,385-394$.

Napoli, E., 2011. PANORMUS User's manual. University of Palermo, Palermo, Italy, pp. 1-74 <www.panormus3d.org>.

Nikuradse, J., 1933. Stromungsgesetze in Rauhen Rohren, VDI-Forsch. 361, 1933 (Eng. trans. 1950. Laws of flow in rough pipes) NACA TM 1292.

Orlandi, P., Leonardi, S., Antonia, R.A., 2006. Turbulent channel flow with either transverse or longitudinal roughness element on one wall. J. Fluid Mech. 561, 279-305.

Piomelli, U., Balaras, E., 2002. Wall-layer model for Large-Eddy Simulation. Annu. Rev. Fluid Mech. 34, 349-374. 
Schultz, M.P., Flack, K.A., 2005. Outer layer similarity in fully rough turbulent boundary layers. Exp. Fluids 38, 328-340.

Schultz, M.P., Flack, K.A., 2009. Turbulent boundary layers on a systematically varied rough wall. Phys. Fluids 21 (015104), 1-9.

Scotti, A., 2006. Direct numerical simulation of turbulent channel flows with boundary roughened with virtual sandpaper. Phys. Fluids 18, 1-4.

Shah, M.K., Agelinchaab, M., Tachie, M.F., 2008. Influence of PIV interrogation area on turbulent statistics up to 4th order moments in smooth and rough wall turbulent flows. Exp. Therm. Fluid Sci. 32, 725-747.

Singh, K.M., Sandham, N.D., Williams, J.J., 2007. Numerical simulation of flow over rough bed. J. Hydraul. Eng. 133 (4), 386-398.

Smalley, R.J., Leonardi, S., Antonia, R.A., Djenidi, L., Orlandi, P., 2002. Reynolds stress anisotropy of turbulent rough wall layers. Exp. Fluids 33, 31-37.

Subramanian, C.S., King, P.I., Reeder, M.F., Ou, S., Rivir, R.B., 2004. Effects of strong irregular roughness on the turbulent boundary layer. Flow Turbul. Combust. 72 349-368.

Tachie, M.F., Bergstrom, D.J., Balachandar, R., 2003. Roughness effects in the low$\operatorname{Re}_{\Theta}$ open-channel turbulent boundary layers. Exp. Fluids 33, 338-346.
Townsend, A.A., 1976. The Structure of Turbulent Shear Flow, second ed. Cambridge University Press.

Volino, R.J., Schultz, M.P., Flack, K.A., 2009. Turbulence structure in a boundary layer with two-dimensional roughness. J. Fluid Mech. 635, 75-101.

Volino, R.J., Schultz, M.P., Flack, K.A., 2011. Turbulence structure in boundary layers over periodic two- and three-dimensional roughness. J. Fluid Mech. 676, 172190.

Wu, Y., Christensen, K.T., 2006. Reynolds-stress enhancement associated with a short fetch of roughness in wall turbulence. AIAA J. 44 (12), 3098-3106.

$\mathrm{Wu}$, Y., Christensen, K.T., 2007. Outer-layer similarity in the presence of a practical rough-wall topography. Phys. Fluids 19, 1-15.

Xie, Z.T., Coceal, O., Castro, I.P., 2008. Large-eddy simulation of flows over random urban-like obstacles. Bound.-Layer Meteorol. 129, 1-23.

Zagarola, M.V., Smits, A.J., 1998. Mean-flow scaling of turbulent pipe flow. J. Fluid Mech. 373, 33-79.

Zang, Y., Street, R.L., Koseff, J.R., 1993. A dynamic mixed subgride-scale model and its application to turbulent recirculating flows. Phys. Fluids 12, 3186-3196. 